\title{
CARACTERÍSTICAS CLÍNICAS E EPIDEMIOLÓGICAS DE 20 PACIENTES PORTADORES DE ESCLEROSE MÚLTIPLA ACOMPANHADOS EM CUIABÁ - MATO GROSSO
}

\author{
Anderson Kuntz Grzesiuk ${ }^{1}$
}

\begin{abstract}
RESUMO - A esclerose múltipla é uma doença imunomediada do sistema nervoso central com ampla variação nos seus aspectos clínicos. No Brasil, aspectos étnicos e demográficos demonstram estas variações, como observado em várias casuísticas brasileiras. O presente estudo retrospectivo consta da análise descritiva dos aspectos clínicos e epidemiológicos de 20 casos de esclerose múltipla acompanhados em Cuiabá, cidade situada na região centro-oeste do Brasil. Os pacientes foram diagnosticados no período entre 1998 e 2005 no ambulatório de Neurologia do CRIDAC. Houve predomínio no sexo feminino (75\%), sendo observada significativa incidência sobre a etnia negra (20\%). A média do EDSS inicial/final foi 1,85/3,9. A forma evolutiva mais comum foi a remitente-recorrente $(75 \%)$, com $55 \%$ do total de pacientes sendo naturais de Mato Grosso. Os sintomas piramidais $(90 \%)$, cerebelares $(60 \%)$ e visuais $(25 \%)$ foram os mais freqüentemente observados. Todos os pacientes estavam em uso de imunomoduladores ou imunossupressores.
\end{abstract}

PALAVRAS-CHAVE: esclerose múltipla, epidemiologia, características clínicas, EDSS.

\begin{abstract}
Clinical and epidemiologic characteristics of 20 patients with multiple sclerosis in Cuiabá - Mato Grosso, Brazil

ABSTRACT - Multiple sclerosis is an acquired immune-mediated disease of the central nervous system that show a widely variation of clinical aspects. In Brazil, demographic and ethnical aspects show these variations as has been demonstrated by many Brazilian papers. This re t rospective study evaluated epidemiological and clinical characteristics of 20 multiple sclerosis patients followed in Cuiaba, situated on Brazil middle west region. The patients were classified followed at Neurology Ambulatory of CRIDAC from 1998 to 2005. The data show a 3:1 female/male ratio and that $20 \%$ of patients were from black origin. EDSS medium range was 3.9; $55 \%$ of patients were born in Mato Grosso State. The most common symptoms were pyramidal $(90 \%)$, cerebellar $(60 \%)$ and visual $(25 \%)$. All patients were on immunomodulatory or immunosupressive treatment.
\end{abstract}

KEY WORDS: multiple sclerosis, epidemiology, clinical findings, EDSS.

A esclerose múltipla (EM) é doença imuno-mediada, inflamatória, caracterizada por repetidos episódios de desmielinização, tendo conseqüentes e variáveis déficits neurológicos causados por lesões focais à mielina. Segundo Victor "situa-se entre as mais veneráveis doenças neurológicas, sendo uma das mais importantes em virtude de sua freqüência, cronicidade e tendência em acometer adultos jovens" ". Esta doença apresenta-se com distribuição unimodal entre os 20-40 anos de idade, com maior incidência no sexo feminino e na etnia branca. Geograficamente incide com maior prevalência nas regiões situadas entre os paralelos 44 e $64 \mathrm{~N}$, sendo consideradas áreas de alta prevalência aquelas com número de casos acima de 30/100000 habitantes, média prevalência áreas com número de casos entre 5 a 30/100000 habitantes e baixa prevalência com número de casos inferior a $5 / 100000$ habitantes ${ }^{1,2}$. O Brasil é considerado um país de baixa prevalência da EM, existindo contudo regiões que apresentam média incidência, como demonstram estudos em cidades como São Paulo ${ }^{3}$, Belo Horizonte $^{4}$ e Botucatu ${ }^{5}$. Especula-se que essa diferença entre as diversas regiões do Brasil decorra em parte de nossa diversidade genética e de nosso índice de miscigenação. Estes fatores, aliados a grande extensão te rritorial do Brasil seriam os responsáveis pela con-

'Neurologista , Ambulatório de Neurologia do Centro de Reabilitação Integral Dom Aquino Corrêa-CRIDAC, Cuiabá MT, Brasil. Recebido 14 Dezembro 2005, recebido na forma final 15 Março 2006. Aceito 2 Maio 2006. 
centração de determinadas características genéticas e fenotípicas em algumas regiões de nosso país. Apesar destes fatores, observações epidemiológicas dos g rupos de estudo do Projeto Atlântico-Sul, Depart amento Científico de Neuroimunologia da Academia Brasileira de Neurologia e do Comitê Brasileiro para Pesquisa e Tratamento da Esclerose Múltipla (BCTRIMS) demonstram que casos de EM são observados na quase totalidade do território brasileiro.

Cuiabá é a capital do Estado de Mato Grosso, situada na região centro-oeste brasileira. Os primórdios desta cidade datam de 1727, sendo denominada sede da província em $1835^{6}$. Cuiabá, assim como Mato $\mathrm{G}$ rosso, permaneceu isolada do restante do país por várias décadas devido a dificuldade de acesso por terra e ao longo trajeto fluvial através da Bacia do Prata, únicos meios de ligação com a então capital Rio de Janeiro. Contudo, a partir dos anos de 1960, o governo federal passou a estimular a migração para a região centro-oeste do Brasil seguindo o lema "Integrar para não entregar", fato que trouxe um grande contingente de pessoas provenientes de todas a regiões do Brasil, principalmente da região sul e sudeste do país, onde predominam brasileiros com descendência européia, de países estes com incidências média e alta de EM. Seguindo esta premissa, passou-se a registrar casos possíveis de EM no Estado de Mato G rosso já nos anos 70, segundo observações feitas por pioneiros das neurociências que aqui chegaram a partir de $1970^{7}$, sendo que os primeiros casos confirmados datam do anos 80 , quando os pacientes tinham seus casos diagnosticados nos grandes centros médicos do Brasil, devido a dificuldade na realização dos exames propedêuticos exigidos pelos critérios da época no Estado de Mato Grosso.

Descreveremos neste artigo as características clínicas e epidemiológicas de 20 pacientes portadores de EM acompanhados no período de 1998 a 2005 no Ambulatório de Neurologia do CRIDAC em Cuiabá, instituição pública que presta atendimento neuro lógico, fisioterápico, fonoaudiológico e psicológico a pacientes oriundos de todas as regiões de Mato Grosso.

\section{MÉTODO}

Realizou-se estudo retrospectivo de 20 casos de EM acompanhados no ambulatório de neurologia do CRIDAC no período entre 1998 e 2005 . Os casos preencheram os critérios diagnósticos de Poser et al. $\left(\mathrm{apud}^{8}\right)$ quando diagnosticados antes de 1998 até o ano de 2001, e os critérios de $\mathrm{McDonald}$ et al. $\left(\right.$ apud $\left.^{8}\right)$ quando diagnosticados após 2001. Classificaram-se os casos nas formas clínicas remitente-recorrente (RR), primária pro g ressiva (PP) e secundária prog ressiva (SP), sendo os pacientes aval iados através de história clínica, exame neurológico, estudos por ressonância magnética (RM) de crânio e RM de medula (quando indicado), estudo do líquor com cálculo do índice de lgG / pesquisa de bandas oligoclonais e sorologias para VDRL, HTLV-1 e HIV.

Os dados clínico-epidemiológicos avaliados foram etnia, sexo, idade, forma evolutiva, EDSS inicial e final, tempo de doença, naturalidade, sintomas clínicos e uso de imunomoduladores ou imunossupressores.

\section{RESULTADOS}

A análise demonstrou que 15 pacientes (75\%) eram do sexo feminino e 5 (25\%) eram do sexo masculino, perfazendo uma relação de 3:1. Dos 20 pacientes, $16(80 \%)$ eram da etnia branca e 4 pacientes (20\%) da etnia negra.

A idade média dos portadores de EM foi 40,2 anos (intenalo 19 e 57 anos), com tempo médio de idade do primeiro surto de 33,8 anos.

O tempo médio de doença foi 6,2 anos (variando entre 1 e 19 anos), ocorrendo 1 óbito no período, em um paciente com a forma PS devido a complicações infecciosas quando em uso de ciclofosfamida.

A forma evolutiva predominante foi a RR com 15 casos (75\%), seguida pela PS com 4 casos (20\%) e pela forma PP com 1 caso $(5 \%)$.

A média do EDSS inicial/final foi $1,85 / 3,9$, com intervalos entre 1,0 e 8,0 pontos. Do total de 20 pacientes, $5(25 \%)$ apresentavam a forma benigna da EM segundo os critérios de Noseworthy et al. ${ }^{9}$.

Os sinais e sintomas clínicos mais freqüentes observados encontram-se dispostos na Tabela 1.

Dos 20 pacientes analisados, 11 (55\%) eram naturais de Mato Grosso e 9 (45\%) eram naturais de outros Estados.

Tabela 1. Sinais e sintomas clínicos observados nos casos de EM avaliados.

\begin{tabular}{lc}
\hline Sinais e sintomas & Número de pacientes \\
\hline Piramidal & 4 \\
Piramidal + ataxia & 8 \\
Piramidal + ataxia + óptico & 2 \\
Piramidal + ataxia + sint. auditivos & 1 \\
Ataxia + óptico & 1 \\
Piramidal + alterações sensitivas & 1 \\
Piramidal + alt. sensitivas + ataxia & 3 \\
Total & 20 \\
\hline
\end{tabular}


Todos os 20 casos apresentaram RM de crânio (sempre) e medula (quando indicado) com imagens compatíveis com patologia desmielinizante, com características sugestivas de EM. As sorologias para HTLV-1, HIV e VDRL foram negativas em todos os casos. Os índices de IgG variaram entre 0,78 e 1,64. As pesquisas de bandas oligoclonais foram realizadas em 11 pacientes, estando presentes no líquor em 3 casos $(27,2 \%)$ e ausentes em 8 casos $(72,8 \%)$. Em 9 pacientes não foi possível a realização da pesquisa de bandas oligoclonais devido a dificuldades na obtenção do referido exame.

O tratamento com imunomoduladores ou imunosupressores adotado nos pacientes até o ano de 2005, de acordo com sua forma evolutiva, encontram-se na Tabela 2.

\section{DISCUSSÃO}

A análise dos dados epidemiológicos deste estudo demonstra predominância da EM no sexo feminino, dado em consonância com a literatura, todavia observamos um índice significativo na etnia negra (20\%), fato incomum na literatura sobre esta patologia. Porém, Alvarenga e col., Oliveira e col. e Ferreira e col. também obtiveram dados semelhantes nesta etnia nas cidades do Rio de Janeiro ${ }^{10}$, São Paulo ${ }^{11}$ e Recife ${ }^{12}$, respectivamente. Ao analisarmos outras séries nacionais, cidades como Curitiba ${ }^{13}$, Belo Horizonte ${ }^{14}$, Santos $^{15}$ e mesmo São Paulo, esta última em estudo de M o reira e col. ${ }^{16}$, demonstraram índices sobre a etnia negra em consonância com a literatura mundial. Particularmente tivemos dificuldade em classificar os portadores quanto a etnia, pois freqüentemente existiam dúvidas nesta classificação quando adotavamse termos como pardos, negros ou mulatos. Observase isto na casuística de Ferreira e col. em Recife ${ }^{12}$, onde a etnia parda foi responsável por $93,2 \%$ dos casos. Em nosso estudo optou-se por incluir pardos e negros em uma mesma classificação.

A idade média de acometimento do primeiro surto da doença ( 33,8 anos), o tempo médio da doença $(6,2$ anos) e a distribuição dos casos entre as formas evolutivas, com predomínio da forma RR, estão em consonância com dados obtidos em outros estudos no Brasil ${ }^{10-13,15,16}$.

No momento de um novo diagnóstico de EM, um fator de angústia tanto para o médico como para o paciente é qual será o prognóstico desta patologia para o referido paciente. Inexistem dados exatos para isto, contudo estudos observacionais de longo prazo p e rmitiram classificar a EM em formas "benigna" e "maligna", consoante o valor evolutivo do EDSS por
Tabela 2. Tipos de tratamento imunomoduladorlimunossu pressor adotados nos casos avaliados de EM.

\begin{tabular}{lcc}
\hline Tratamento & Forma evolutiva & Casos \\
\hline Metotrexate & PP & 1 \\
Interferon beta 1-b & SP & 2 \\
Interferon beta 1-a & RR & 15 \\
Mitoxantrone & SP & 1 \\
Ciclofosfamida & SP & 1 * \\
Total de casos & & 20 \\
\hline *óbito em 2004
\end{tabular}

*óbito em 2004.

determinado tempo, sendo na forma benigna um valor de EDSS igual ou menor que 3,5 em 10 anos $^{9} \mathrm{~A}$ expectativa é de encontrar-se pacientes com a form a "benigna" em até $20 \%$ dos casos. Séries nacionais tiveram este índice variando entre $7,0 \%$ e $19,8 \% 12,13$. O valor encontrado em nossa série $(25 \%)$ apresentase acima do esperado, contudo este dado está baseado em uma amostragem pequena, não podendo servir como parâmetro para a região, pois inexistem dados sobre esta doença em nosso Estado. Chamou-nos a atenção o fato de que dentre os 11 pacientes em que foi possível a realização de pesquisa de banda oligoclonal, $72,8 \%$ destes terem o resultado negativo, apesar do índice quantitativo estar acima de 0,73 (valor de corte). Não há dados para concluirmos se tratou-se de falha no armazenamento da amostra, uma vez que o exame é encaminhado para processamento fora do Estado, dificuldades técnicas na manufatura do mesmo, ou se existem fatores que possam sugerir que a EM em nosso Estado tenha um comportamento mais benigno, um vez que existem evidências de que pacientes com bandas oligoclonais negativas tenham melhor prognóstico ${ }^{17}$. Talvez as características genotípicas e fenotípicas de nossa região possam estar relacionadas a este achado em alguma proporção uma vez que, ao analisarmos os dados disponíveis sobre a EM no Brasil, podemos inferir que o comportamento desta doença é diverso daquele encontrado em outros países.

O Estado de Mato Grosso, por tratar-se ainda de uma região em expansão comercial e industrial, apresenta algumas particularidades, como o fato de os a p a relhos para realização de ressonância magnética e os laboratórios para a realização de estudos quantitativos do liquor estarem situados na capital Cuiabá. Estes fatores acabam por referenciar os casos com suspeita de doença desmielinizante para Cuiabá, onde poderão ter seu diagnóstico confirmado e encaminhados para tratamento específico. 
O presente estudo merece críticas, seja pelo núme ropequeno da amostragem, seja por tratar-se de método retrospectivo, contudo espera-se contribuir para que a realidade da EM em Cuiabá e Mato Grosso possa ser conhecida no futuro através de estudos de incidência e prevalência. Para tanto, torna-se mister a criação de um centro de referência para tratamento da EM na capital Cuiabá, para que assim o ciclo atendimento neurológico/diagnóstico/tratamento/seguimento possa demonstrar as reais idiossincrasias da EM em Mato Grosso.

\section{REFERÊNCIAS}

1. Victor M, Ropper AH. M. Multiple sclerosis and allied demyelinative diseases. In Victor M (ed). Adams and Victor's Principles of Neurology, $7^{\text {th }}$ Edition-International edition, New York: McGraw-Hill,2001:955.

2. CallegaroD. Aspectos epidemiológicos. In Tilbery CP (ed). Esclerose múltipla no Brasil: aspectos clínicos e terapêuticos. São Paulo: Atheneu, 2005:13-15.

3. Callegaro D, Goldbaum M, Morais L, et al. The prevalence of multiple sclerosis in the city of São Paulo, Brazil. Acta Neurol Scand 2001;104:208213.

4. Lana-Peixoto MA, Frota E, Campos GB, Botelho CM, Aragão AL. The p revalence of multiple sclerosis in Belo Horizonte, Brazil. Multiple Sclerosis 2002;8(Suppl):S38.

5. Rocha FC, Herrera LC, Morales RR. Multiple sclerosis in Botucatu, Brazil: a population study. Multiple Sclerosis 2002;8(Suppl):S41.
6. Costa MF. História de um país inexistente: o Pantanal entre os séculos XVI e XVIII. São Paulo: Editora Estação Liberdade,1999:54-56.

7. Silva NM. História da neurologia em Mato Grosso. In Reimão R (org). História da neurologia no Brasil. São Paulo: Lemos Editorial;1999: 133-138.

8. Blumhardt LD. Dictionary of multiple sclerosis. London: Martin Dunitz, 2004:47-51.

9. Noseworthy JH, Lucchinetti C, Rodriguez M, Weinshenker BG. Multiple sclerosis. N Engl J Med 2000;343:938-951.

10. Papais-Alvarenga RM, Santos CMM, Abreu JS, et al. Esclerose múltipla: perfil clínico e evolutivo no Rio de Janeiro. Rev Bras Neurol 1995;31:7587.

11. Oliveira EML, Annes M, Oliveira ASB, Gabbai AA. Esclerose múltipla: estudo clínico de 50 pacientes acompanhados no Ambulatório de Neurologia UNIFESP-EPM. Arq Neuropsiquiatr 1999;57:51-55.

12. Fer reira MLB, Machado MI, Vilela ML, et al. Epidemiologia de 118 casos de esclerose múltipla com seguimento de 15 anos no centro de referência do Hospital da Restauração de Pernambuco. A rq Neuropsiquiatr 2004;62:1027-1032.

13. Arruda WO, Scola RH, Teive HAG, Werneck LC. Multiple sclerosis: report on 200 cases from Curitiba, Southern Brazil and comparison with other Brazilian series. Arq Neuropsiquiatr 2001;59:165-170.

14. Lana-Peixoto MA, Lana-Peixoto MI. Is multiple sclerosis in Brazil and Asia alike? Arq Neuropsiquiatr 2000;58:467-470.

15. Fragoso YD, Fiore APP. Description and characteristics of 81 patients attending the reference center for multiple sclerosis of the coastal region of the State of Sao Paulo-Brazil. Arq Neuropsiquiatr 2005;63:741-744.

16. Mo reira MA, Felipe E, Mendes MF, Tilbery CP. Esclerose múltipla: estudo descritivo de suas formas clínicas em 302 casos. A rq Neuropsiquiatr 2000;58:460-466.

17. Sá MJ, Sequeira L, Rio ME, Thompson EJ. Oligoclonal IgG bands in the cerebrospinal fluid of Portuguese patients with multiple sclerosis. Arq Neuropsiquiatr 2005;63:375-379. 